\title{
Sixteen weeks of resistance training can decrease the risk of metabolic syndrome in healthy postmenopausal women
}

This article was published in the following Dove Press journal:

Clinical Interventions in Aging

13 September 2013

Number of times this article has been viewed

\author{
Miguel Soares Conceição' \\ Valéria Bonganha' \\ Felipe Cassaro Vechin ${ }^{2}$ \\ Ricardo Paes de Barros \\ Berton' \\ Manoel Emílio Lixandrão' \\ Felipe Romano Damas \\ Nogueira' \\ Giovana Vergínia de Souza' \\ Mara Patricia Traina \\ Chacon-Mikahil' \\ Cleiton Augusto Libardi
}

'Exercise Physiology Laboratory, School of Physical Education, State University of Campinas, Campinas, ${ }^{2}$ Laboratory of Neuromuscular Adaptation to Strength Training, School of Physical Education and Sport, University of São Paulo, São Paulo, Brazil
Correspondence: Miguel Soares Conceição Exercise Physiology Laboratory, School of Physical Education, State University of Campinas, Av Érico Veríssimo 70I, |3083-85I, PO Box 6134, Campinas, Brazil

Tel +551935216625

$\mathrm{Fax}+551935216600$

Email conceicao.miguel0106@gmail.com
Background: The postmenopausal phase has been considered an aggravating factor for developing metabolic syndrome. Notwithstanding, no studies have as yet investigated the effects of resistance training on metabolic syndrome in postmenopausal women. Thus, the purpose of this study was to verify whether resistance training could reduce the risk of metabolic syndrome in postmenopausal women.

Methods: Twenty postmenopausal women were randomly assigned to a resistance training protocol $(n=10,53.40 \pm 3.95$ years, $64.58 \pm 9.22 \mathrm{~kg})$ or a control group $(\mathrm{n}=10,53.0 \pm 5.7$ years, $64.03 \pm 5.03 \mathrm{~kg}$ ). In the resistance training protocol, ten exercises were performed, with $3 \times 8-10$ maximal repetitions three times per week, and the load was increased every week. Twoway analysis of variance was used to evaluate specific metabolic syndrome Z-score, high density lipoprotein cholesterol, fasting blood glucose, triglycerides, waist circumference, blood pressure, strength, and body composition. The level of statistical significance was set at $P<0.05$.

Results: The main results demonstrated a significant decrease of metabolic syndrome Z-score when the postmenopausal women performed resistance training $(P=0.0162)$. Moreover, we observed decreases in fasting blood glucose for the resistance training group $(P=0.001)$, and also significant improvements in lean body mass $(P=0.042,2.46 \%)$, reduction of body fat percentage $(P=0.001,-6.75 \%)$ and noticeable increases in muscle strength after resistance training to leg press $(P=0.004,41.29 \%)$ and bench press $(P=0.0001,27.23 \%)$.

Conclusion: It was concluded that resistance training performed three times a week may reduce the metabolic syndrome Z-score with concomitant decreases in fasting blood glucose, improvements in body composition, and muscle strength in postmenopausal women.

Keywords: metabolic syndrome, resistance training, postmenopausal women

\section{Introduction}

The prevalence of metabolic syndrome has increased in recent decades, mainly in developing countries. ${ }^{1}$ In Brazil, more than $30 \%$ of middle-aged adults are classified as having metabolic syndrome, and it has been shown that this syndrome predominantly affects women. ${ }^{1}$ Nevertheless, the prevalence of metabolic syndrome may vary according to gender, age, and the diagnostic criteria used..$^{2-4}$ There is a higher incidence of metabolic syndrome in the postmenopausal period, especially in middle-aged women, who after menopause undergo significant adverse changes in body composition, including loss of lean body mass, increases in fat body mass, and redistribution of fat from the periphery to the central region of the body. ${ }^{5-10}$ Other related modifications associated with menopause, such as increased blood pressure, reductions in glucose tolerance, atherogenic changes in the lipid and coagulation profiles, and impairment 
of endothelial function, are common and can also increase the incidence of metabolic syndrome..$^{411-16}$ Further, the postmenopausal phase can induce severe reductions in strength and muscle mass (ie, sarcopenia) especially among sedentary women. ${ }^{8,17}$ These facts provide insights showing that the postmenopausal period itself is an independent risk factor for metabolic syndrome, ${ }^{17,18}$ increasing the chances of developing cardiovascular disease and consequently premature death. ${ }^{19-22}$ Therefore, it is necessary to develop strategies to reduce the risk of metabolic syndrome. It has been proposed that incorporation of daily physical exercise might be an effective strategy against development of metabolic syndrome. ${ }^{23,24}$

Several studies have demonstrated the beneficial effects of aerobic training on the comorbidity of obesity and other cardiometabolic risk factors such as metabolic syndrome. ${ }^{21,25,26}$ Johnson et $\mathrm{al}^{25}$ using 80 women (all postmenopausal) and 91 men with a sedentary lifestyle, aged between 40 and 65 years, showed that aerobic exercise of moderate intensity results in a significant reduction in the risk of metabolic syndrome.

On the other hand, resistance training induces differential benefits when compared with aerobic training, such as increases in muscle and bone mass, strength development, and improvement in functional capacity. ${ }^{27-29}$ Thus, these benefits observed after a resistance training intervention can prevent and/or amend sarcopenia and other related comorbidities. ${ }^{20,30}$ In addition, resistance training has been demonstrated to ameliorate the lipid profile ${ }^{31}$ and diminish intra-abdominal adipose tissue. ${ }^{32}$ Despite resistance training provides several benefits concerning different metabolic syndrome variables, few studies have investigated the beneficial impacts of resistance training on reduction of metabolic syndrome in postmenopausal women, who seem to be more susceptible to developing these risk factors. Therefore, the purpose of the present study was to investigate if resistance training itself is capable of reducing risk factors for metabolic syndrome in the postmenopausal years. It was hypothesized that resistance training could decrease this risk in postmenopausal women.

\section{Materials and methods Subjects}

The experimental protocol was approved by the research ethics committee of the State University of Campinas. Thereafter, the subjects were recruited through newspaper, magazine, and Internet advertisements. Potential subjects completed a preliminary medical history, an exercise questionnaire, and all participants were informed of the purpose and risks of the study before signing their informed consent. Also, before inclusion in the study, a complete medical examination was carried out and subjects were excluded if acute illness, severe hypertension, insulin-dependent diabetes, or myocardial infarction were diagnosed, or if they were taking medication known to affect plasma lipoprotein levels, had diabetes mellitus, or were on hormone replacement therapy. All women participating in the study were in the postmenopausal period ( $>12$ months of amenorrhea), middle-aged (aged 45-60 years), and considered to have a sedentary lifestyle without any consistent exercise in the previous year. Sedentary lifestyle was measured and analyzed using the Baecke questionnaire on physical activity. ${ }^{33}$

A sample size estimation was conducted using $G^{*}$ Power (version 3.0.10, Universitat Kiel, Germany). Previous study data from our laboratory ${ }^{31}$, which compared the lipid profile (total cholesterol) between men and women, in which resistance training was the exercise model of intervention, were utilized to perform the sample size estimation. We based the calculation on an effect size of 1 , an $\alpha$ level of 0.05 , and a power $(1-\beta)$ of 0.80 . Sample size estimation showed that it would be necessary to include at least 14 volunteers (seven subjects per group). Therefore, we recruited 20 volunteers who were randomly assigned into two groups: a resistance training group $(n=10$; mean age $53.40 \pm 3.95$ years; body mass $64.58 \pm 9.22 \mathrm{~kg}$; height $1.57 \pm 0.06 \mathrm{~m}$; body mass index $26.22 \pm 3.26 \mathrm{~kg} / \mathrm{m}^{2}$; and $5.50 \pm 3.21$ years into the postmenopausal phase) and a control group $(\mathrm{n}=10$; mean age $53.0 \pm 5.7$ years; body mass $64.03 \pm 5.03 \mathrm{~kg}$; height $1.60 \pm 0.05 \mathrm{~m}$; body mass index $25.30 \pm 1.81 \mathrm{~kg} / \mathrm{m}^{2}$; and $7.46 \pm 6.01$ years into the postmenopausal phase) who did not perform any kind of exercise during the experimental period.

\section{Experimental design}

All subjects underwent three visits before beginning training. On the first visit, blood samples, anthropometric assessments, blood pressure, and familiarization with the training exercises were undertaken. On the following visit, the first maximal strength test session was performed. After 72 hours, a second maximal strength test was performed and the value obtained was used in this study, only for strength assessment. The resistance training group trained throughout 16 weeks, while the control group did not perform any kind of physical exercise. All assessments were performed again at the end of the experimental period for both study groups. To avoid circadian variations, all evaluations were done at the same time of day (between 8 am and $10 \mathrm{am}$ ), with controlled temperature conditions $\left(22^{\circ} \mathrm{C}\right)$, at the same relative air humidity (about 60\%) and atmospheric pressure. No dietary 
prescriptions were provided to the participants. However, they were all advised to maintain their normal alimentary habits throughout the study period as well as to maintain their normal daily activities.

\section{Z-score analyses}

The metabolic syndrome Z-score was used in the present study as a continuous score of the five metabolic syndrome variables,${ }^{30}$ in agreement with the Adult Treatment Panel III criteria. A Z-score was calculated for each variable using individual subject data, and standard deviations of data for the entire group at baseline ( $n=52$, number of subjects recruited into the study at the beginning). Gender-specific metabolic syndrome Z-score equations were used following the Adult Treatment Panel III criteria. The equation used to calculate the metabolic syndrome $\mathrm{Z}$-score ${ }^{30}$ was as follows:

$$
\begin{aligned}
\mathrm{Z} \text {-score }= & {[(50-\mathrm{HDL}) / 14.1+(\mathrm{TG}-150) / 90.1] } \\
& +[(\text { fasting blood glucose }-100) / 11.3] \\
& +[(\text { waist circumference }-88) / 7] \\
& +[(\text { mean arterial pressure }-100) / 14.1],
\end{aligned}
$$

where HDL is high-density lipoprotein and TG is triglycerides.

\section{Serum lipid profile and glucose}

Resting venous blood samples were collected via venipuncture from an antecubital forearm vein at 8 am after a 12-hour overnight fast and abstinence from exercise for 24 hours. The samples were centrifuged at $3000 \mathrm{rpm}$ for 15 minutes. The resulting serum was then removed and stored at $-70^{\circ} \mathrm{C}$ until subsequent analysis. Serum concentrations of glucose, TG, and HDL cholesterol were assessed using an automatic analyzer (RA 1000, Technicon Corporation, Tarrytown, NY, USA) and a commercially available kit. The intra-assay variability, interassay variability, and sensitivity were, respectively, $1.08 \%, 1.82 \%$, and $1.8 \mathrm{mg} / \mathrm{dL}$ for glucose; $1.5 \%, 1.8 \%$, and $4 \mathrm{mg} / \mathrm{dL}$ for TG; and $0.89 \%, 2.08 \%$, and $3 \mathrm{mg} / \mathrm{dL}$ for HDL cholesterol.

\section{Anthropometric variables}

Subject body weight was measured on a balance scale (Filizola, São Paulo, Brazil) calibrated to the nearest $0.1 \mathrm{~kg}$. Barefoot standing height was measured to the nearest $0.1 \mathrm{~cm}$ using a wall-mounted stadiometer. Waist circumference was measured using a constant tension tape at the midpoint between the lowest rib and the iliac crest. Body density was estimated using the skin-fold procedure according to Jackson et al, ${ }^{34}$ using a skinfold caliper (Lange, Beta Technology, Santa Cruz, CA, USA) at the chest, abdomen, thigh, triceps, subscapular, suprailiac, and mid axillary points. The percentage of body fat was obtained from body density using the Siri equation..$^{35}$ All assessments were performed by the same professional.

\section{Mean blood pressure}

Blood pressure assessments were done after approximately 10 minutes of rest using a mercury sphygmomanometer and stethoscope. The women were positioned supine and the same professional made the measurements. All measurements were taken in duplicate and the mean of two assessments was used. The formula for mean blood pressure (MBP) assessment was:

$$
\mathrm{MBP}=\mathrm{DBP}+[0.333 \times(\mathrm{SBP}-\mathrm{DBP})],
$$

where DBP is diastolic blood pressure and SBP is systolic blood pressure.

\section{Maximal strength assessments}

Muscle strength was assessed by one maximal repetition test for bench and leg press exercises. Individuals were required to perform eight repetitions at $50 \%$ of one repetition maximum, estimated according to each participant's capacity, to rest for 1 minute, and then perform a further three repetitions at $70 \%$ of one repetition maximum. After a 3-minute rest, subsequent trials were performed for one repetition with progressively heavier weights until the one repetition maximum was determined within three attempts, with 3-5 minutes of rest between trials. Standardization of range of motion and performance of the exercises was done according to Libardi et al. ${ }^{31}$ Before the beginning of the study, participants performed two familiarization trial sessions composed of two sets of moderate intensity exercises, with 72 hours of rest between them. The purpose of this familiarization was to reduce learning effects.

\section{Resistance training}

The subjects performed 16 weeks of resistance training with three training sessions per week on alternate days (Mondays, Wednesdays, and Fridays). During the first 8-week period, subjects performed leg press, leg extension, leg curl, bench press, lat pulldown, lateral raise, triceps pushdown, arm curl, and basic abdominal crunch protocols. The training consisted of three sets of ten repetition maxima, with a 60 -second rest between sets. After 8 weeks, the exercises were maintained; however, the subjects performed eight repetition maxima with a 90 -second rest between sets. ${ }^{27}$ The resistance training was based on the linear periodization or "classic" model, based on volume and intensity change during mesocycles 
( 8 weeks). It is characterized by high volume and low intensity in the initial training period and progresses to the extent that the volume is lower than the intensity, ie, the intensity increases and the volume decreases. ${ }^{36}$ The numbers of repetitions were reduced (maintaining the minimal zone established for each cycle) as the intensity increased.

The workloads were adjusted weekly according to Libardi et al. ${ }^{29}$ The participants were encouraged to perform the greatest number of repetitions they had achieved in the previous training session when they came to the last set of each exercise, maintaining the same range of motion and execution velocity as previously determined. Workloads were increased by $1 \mathrm{~kg}$ for the lower body and $0.5 \mathrm{~kg}$ for the upper body for each repetition performed over the established training protocol, according to the greatest number of repetitions accomplished by the participants in the previous week.

\section{Statistical analyses}

Statistical analyses were performed using Statistica version 6.1 software (StatSoft Inc., Tulsa, OK, USA). First, a Shapiro-Wilk test was used to test the normality of the data. A two-way repeated-measures analysis of variance was used to evaluate within/between-group changes in metabolic syndrome Z-score, metabolic syndrome variables, and muscle strength, with groups and time set as independent variables and subjects set as a random factor. When a significant $F$ was obtained, the Tukey post hoc test was used to identify significant differences. The effect sizes were classified as very large $(<-2.0)$, large $(-2.0$ to -1.2$)$, moderate $(-1.2$ to -0.6$)$, small $(-0.6$ to $-0.2)$, trivial ( -0.2 to 0.2$)$, small $(0.2$ to 0.6$)$, moderate ( 0.6 to $1.2)$, large (1.2 to 2.0$)$, and very large $(>2.0)$. The level of statistical significance was set at $P<0.05$. Data are presented as the mean \pm standard deviation unless otherwise stated.

\section{Results}

\section{Metabolic syndrome Z-score}

Metabolic syndrome Z-score values are shown in Figure 1. Only resistance training significantly reduced the metabolic syndrome Z-score after 16 weeks $(P=0.0162)$.

\section{Risk factors for metabolic syndrome}

The risk factors for metabolic syndrome are presented in Table 1. A significant group versus time interaction for HDL cholesterol $(F=7.35, P=0.014,13.20 \%)$, waist circumference $(F=5.79, P=0.027,-0.25 \%)$ and glucose $(F=9.75$, $P=0.006,-13.97 \%$ ) was observed, with the post hoc test demonstrating that resistance training achieved a significant glucose reduction after 16 weeks $(P=0.001)$. However, the

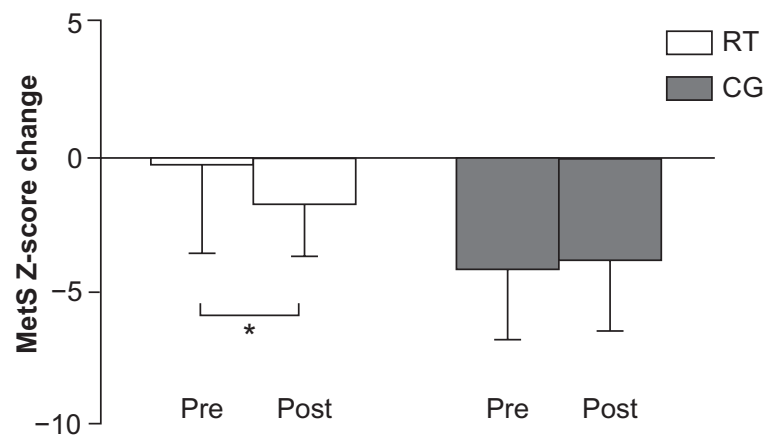

Figure I Comparison between the effects of resistance training $(n=10)$ and control group $(\mathrm{n}=10)$ on changes in metabolic syndrome Z-score.

Note: *Significant difference $(P<0.05)$ between pre- and post- resistance training. Abbreviations: MetS, metabolic syndrome; CG, control group; RT, resistance training group; Pre, baseline; Post, after 16 weeks intervention period.

post hoc test was not able to find exact differences for HDL cholesterol and waist circumference $(P>0.05)$. Further, there were no significant changes in $\mathrm{TG}$ and blood pressure (SBP, DBP, and MBP) in either of the groups.

\section{Body composition and muscle strength}

Table 2 shows the results for body composition and muscle strength. Regarding changes in body composition, we found a significant group versus time interaction for percentage of body fat $(F=16.75, P=0.001,-6.75 \%)$, lean body mass $(F=4.523, P=0.042,2.46 \%)$, and fat body mass $(F=16.58$, $P=0.001,-7.06 \%)$. The post hoc test showed that resistance training significantly decreased body fat percentage and fat body mass ( $P=0.001$ and $P=0.004$, respectively) after training compared with the control group. Further, we found an increase in lean body mass after resistance training $(P=0.042)$ compared with the control group. There were no changes in either group for body mass and body mass index, and no significant changes in body composition were found in the control group. Regarding muscle strength, we observed a significant group versus time interaction for leg press ( $F=10.60, P=0.004,41.29 \%)$ and bench press $(F=25.47$, $P=0.000,27.23 \%$ ), with the post hoc test demonstrating that the resistance training group increased strength for leg press and bench press after a 16-week period $(P<0.001$ for both exercises). There was no significant improvement of muscle strength in the control group after 16 weeks.

\section{Discussion}

The purpose of the present study was to investigate if resistance training can help to reduce the risk of metabolic syndrome in healthy postmenopausal women. The results showed a decrease in metabolic syndrome Z-score values, 
Table I Metabolic syndrome components at baseline (pre) and after the 16-week intervention (post) period

\begin{tabular}{|c|c|c|c|c|c|c|c|c|}
\hline \multirow[t]{2}{*}{ Variables } & \multicolumn{2}{|l|}{ Control group } & \multirow[t]{2}{*}{$\Delta \%$} & \multirow[t]{2}{*}{ ES } & \multicolumn{2}{|c|}{ Resistance training group } & \multirow[t]{2}{*}{$\Delta \%$} & \multirow[t]{2}{*}{ ES } \\
\hline & Pre & Post & & & Pre & Post & & \\
\hline HDL-C (mmol/L) & $54.72 \pm 8.21$ & $52.00 \pm 7.24$ & -4.96 & S & $50.92 \pm 10.09$ & $57.64 \pm 12.70$ & 13.20 & $S$ \\
\hline Glucose (mmol/L) & $93.99 \pm 7.84$ & $93.10 \pm 7.55$ & -0.95 & $\mathrm{~T}$ & $98.74 \pm|3.4|$ & $84.94 \pm 6.33^{*}$ & -13.97 & L \\
\hline Triglycerides (mmol/L) & $109.21 \pm 62.07$ & $117.80 \pm 47.13$ & 7.87 & $\mathrm{~T}$ & $191.28 \pm 75.98$ & $232.31 \pm 77.98$ & 21.45 & $S$ \\
\hline$W C(\mathrm{~cm})$ & $77.54 \pm 3.67$ & $78.96 \pm 4.24$ & 1.81 & S & $81.53 \pm 10.51$ & $81.22 \pm 9.64$ & -0.25 & $\mathrm{~T}$ \\
\hline $\mathrm{DBP}(\mathrm{mmHg})$ & $78.40 \pm 6.50$ & $78.00 \pm 7.70$ & -0.44 & $\mathrm{~T}$ & $89.80 \pm 7.27$ & $88.20 \pm 5.45$ & -1.60 & S \\
\hline $\mathrm{SBP}(\mathrm{mmHg})$ & $111.80 \pm 7.30$ & $\mathrm{I} \mid 3.30 \pm 8.40$ & 1.56 & $\mathrm{~T}$ & $138.40 \pm 15.37$ & $130.80 \pm 16.06$ & -7.60 & $S$ \\
\hline MBP $(\mathrm{mmHg})$ & $80.59 \pm 28.94$ & $80.79 \pm 29.20$ & 0.25 & $\mathrm{~T}$ & $105.98 \pm 6.67$ & $102.39 \pm 6.73$ & -3.39 & $S$ \\
\hline
\end{tabular}

Notes: *Significant difference $(P<0.05)$ between pre and post. Pre represents baseline values, post represents values after the 16 -weeks intervention period. Pre and Post figures are shown as means \pm standard deviations.

Abbreviations: ES, effect size; T, trivial; S, small; L, large; HDL-C, high density lipoprotein cholesterol; WC, waist circumference; MBP, mean blood pressure; SBP, systolic blood pressure; DBP, diastolic blood pressure.

so we can infer that resistance training is efficient in helping to decrease the risk of metabolic syndrome in middle-aged postmenopausal women. Further, the resistance training group showed significant decreases in fasting glucose levels and fat mass, and also increases in lean body mass and muscle strength.

Table 2 shows muscle strength before and after the intervention period. We observed significant improvements in leg press (41.29\%) and bench press (27.23\%) muscle strength for the resistance training group, but no changes were observed in the control group. Orsatti et $\mathrm{al}^{30}$ demonstrated similar enhancement in muscle strength on bench press $(29.5 \%)$ after 16 weeks of training. The similarity of these results could be attributed to the training model used, ie, resistance training based on a series of maximal repetitions. The resistance training method used in the present study and in the study realized by Orsatti et $\mathrm{al}^{30}$ has been demonstrated to be the optimum method for improving strength and lean body mass. ${ }^{37}$ Increased muscle strength seems to be extremely important in the prevention of metabolic syndrome. ${ }^{38,39}$ Also, our results for muscle strength confirm the efficacy of our training protocol and corroborate the findings of Wijndaele et al. ${ }^{39}$ These authors, in a cross-sectional study including
1,019 (571 men) adults aged 18-75 years demonstrated that the risk of metabolic syndrome was inversely associated with muscle strength, independently of aerobic fitness and dietary intake, suggesting a possible protective effect of muscle strength in metabolic syndrome. Our results are also consistent with those of Wijndaele et al, ${ }^{39}$ showing that resistance training improves muscle strength and can induce positive modifications in body composition.

After menopause, women are likely to experience significant changes in body composition, including increased body fat and decreased lean body mass (ie, sarcopenia) $)^{6,9,10,40}$ and these changes are related to several diseases..$^{20,30,37}$ Like Johnston et $\mathrm{al}^{41}$ and Orsatti et al, ${ }^{30}$ we noted a significant increase in lean body mass $(2.46 \%)$ after a resistance training intervention (Table 2). Orsatti et $\mathrm{al}^{30}$ observed a significant increase in muscle mass (about 10\%) in sedentary postmenopausal women after 16 weeks of resistance training comprising three sessions per week. Although Orsatti et $\mathrm{al}^{30}$ did not show a decrease in fat mass, our protocol was effective in decreasing percent body fat (by about 6.75\%) and fat body mass (by about $1.56 \mathrm{~kg}$ ). Although the skin-fold method is not a gold standard for assessing body composition and, thus, configures a limitation of the present study, previous work has shown its

Table 2 Body composition and muscle strength at baseline (pre) and after the 16 week intervention (post) period

\begin{tabular}{|c|c|c|c|c|c|c|}
\hline \multirow[t]{2}{*}{ Variables } & \multicolumn{2}{|l|}{ Control group } & \multirow[t]{2}{*}{$\Delta \%$} & \multicolumn{2}{|c|}{ Resistance training group } & \multirow[t]{2}{*}{$\Delta \%$} \\
\hline & Pre & Post & & Pre & Post & \\
\hline BM (kg) & $64.25 \pm 5.03$ & $64.90 \pm 5.13$ & 1.01 & $64.58 \pm 9.22$ & $64.07 \pm 9.18$ & -0.79 \\
\hline BMI $\left(k g / m^{2}\right)$ & $25.26 \pm 1.81$ & $25.53 \pm 2.11$ & 1.07 & $26.22 \pm 3.26$ & $26.00 \pm 3.11$ & -0.083 \\
\hline$\%$ body fat & $35.80 \pm 5.71$ & $36.58 \pm 4.61$ & 2.15 & $33.68 \pm 3.93$ & $31.4 I \pm 4.86^{*}$ & -6.75 \\
\hline Lean body mass (kg) & $41.05 \pm 2.43$ & $41.01 \pm 2.49$ & -0.09 & $42.54 \pm 4.23$ & $43.59 \pm 3.93^{*}$ & 2.46 \\
\hline Fat body mass (kg) & $23.20 \pm 4.95$ & $23.88 \pm 4.37$ & 2.93 & $22.04 \pm 5.35$ & $20.48 \pm 5.66^{*}$ & -7.06 \\
\hline Bench press (kg) & $35.50 \pm 5.13$ & $37.40 \pm 6.60$ & 5.35 & $38.20 \pm 7.89$ & $48.60 \pm 7.66^{*}$ & 27.23 \\
\hline Leg press (kg) & $134.00 \pm 24.29$ & $150.60 \pm 21.89$ & 12.39 & $|28.60 \pm| 2.7 \mid$ & $181.70 \pm 42.98 *$ & 41.29 \\
\hline
\end{tabular}

Notes: *Significant difference $(P<0.05)$ between pre and post. Pre represents baseline values, post represents values after the 16 -weeks intervention period. Pre and Post figures are shown as means \pm standard deviations.

Abbreviations: BM, body mass; BMI, body mass index. 
validity compared to the radiographic technique of computerized tomography and magnetic resonance imaging. ${ }^{42,43}$

The increase in lean body mass is important for protection against sarcopenia, and can be related to improved glucose uptake. Although our sample had normal glucose levels, improved glucose intake can also be associated with qualitative changes in resistance-trained muscle. ${ }^{22,44}$ Holten et $\mathrm{a}^{44}$ showed that, after 6 weeks of resistance training, insulin action in skeletal muscle was enhanced in both type 2 diabetics and healthy subjects. Consequently, the authors concluded that increased muscle glucose transporter isoform- 4 content and expression and/or activity of various insulin signaling proteins are part of the mechanism underlying the improvement in insulin action. Therefore, we can speculate that the improvement in glucose control achieved in postmenopausal women during the present study (Table 1) could be due to both increased lean body mass and/or functional improvements in resistance-trained muscle, which has been shown to be important for this population. Further, decreased fasting blood glucose and improved body composition may also contribute to decreasing the risk of metabolic syndrome.

Taking into account the concept of the Z-score (ie, a standard score which indicates by how much the standard deviation, an observation, or datum point is above or below the mean), we can infer that resistance training was effective in improving the Z-score (see Figure 1) because the continuous score would be more sensitive to both small and large changes. ${ }^{25}$ Hence, the decrease in fasting blood glucose levels coupled with the small modifications in blood pressure and HDL cholesterol (see effect size, Table 1) observed in the resistance training group after the intervention period could have contributed to the decrease in metabolic syndrome Z-score (Figure 1). On the other hand, we did not show a group versus time interaction for waist circumference, HDL cholesterol, or blood pressure. These results are probably due to the fact that our sample initially had normal levels for these parameters. Further, changes in HDL cholesterol, blood pressure, and waist circumference are more favorably modified when dynamic endurance training is performed. ${ }^{45}$

Potteiger et $\mathrm{al}^{46}$ undertook 6 months of resistance training in 35 overweight males, and showed that resistance training was effective in reducing the risk of cardiovascular disease by positively affecting waist circumference and mean arterial blood pressure, and by decreasing body fat, fat mass, fasting insulin, and insulin resistance. Nevertheless, the authors proposed both resistance training and dietary energy restriction. Even with these independent results, the Z-score did not show a significant positive change, in contrast with our results, probably because the modifications in metabolic syndrome Z-score were moderate. The present study did not propose any nutritional changes, but analyses of the Z-score showed an important reduction in risk of metabolic syndrome for the resistance training group in comparison to the control group. Future studies using a resistance training protocol combined with dietary energy restriction in postmenopausal women could be performed in order to produce greater modifications in Z-score metabolic syndrome risk.

In summary, we conclude that resistance training is effective for reducing the risk of metabolic syndrome, given that resistance training helped in decreasing $Z$-score values in middle-aged postmenopausal women. In addition, resistance training was effective for increasing muscle strength in the upper and lower limbs, increasing lean body mass, and decreasing body fat and fasting blood glucose, which are desirable improvements for the health of postmenopausal women.

\section{Acknowledgment}

The corresponding author would like to express gratitude to São Paulo Research Foundation (FAPESP) for financial support (2012/09709-0).

\section{Disclosure}

The authors declare that they have no conflicts of interest in this work.

\section{References}

1. Dutra ES, de Carvalho KM, Miyazaki E, Hamann EM, Ito MK. Metabolic syndrome in central Brazil: prevalence and correlates in the adult population. Diabetol Metab Syndr. 2012;4:20.

2. Expert Panel on Detection, Evaluation, and Treatment of High Blood Cholesterol in Adults. Executive Summary of the Third Report of the National Cholesterol Education Program (NCEP) Expert Panel on Detection, Evaluation, and Treatment of High Blood Cholesterol in Adults (Adult Treatment Panel III). JAMA. 2001;285:2486-2497.

3. Cornier MA, Dabelea D, Hernandez TL, et al. The metabolic syndrome. Endocr Rev. 2008;29:777-822.

4. Spencer CP, Godsland IF, Stevenson JC. Is there a menopausal metabolic syndrome? Gynecol Endocrinol. 1997;11:341-355.

5. Douchi T, Yamamoto S, Nakamura S, et al. The effect of menopause on regional and total body lean mass. Maturitas. 1998;29:247-252.

6. Douchi T, Yamamoto S, Yoshimitsu N, Andoh T, Matsuo T, Nagata Y. Relative contribution of aging and menopause to changes in lean and fat mass in segmental regions. Maturitas. 2002;42:301-306.

7. Ley CJ, Lees B, Stevenson JC. Sex- and menopause-associated changes in body-fat distribution. Am J Clin Nutr. 1992;55:950-954.

8. Svendsen OL, Hassager C, Christiansen C. Age- and menopauseassociated variations in body composition and fat distribution in healthy women as measured by dual-energy X-ray absorptiometry. Metabolism. 1995;44:369-373.

9. Toth MJ, Tchernof A, Sites CK, Poehlman ET. Effect of menopausal status on body composition and abdominal fat distribution. Int J Obes Relat Metab Disord. 2000;24:226-231. 
10. Trémollieres FA, Pouilles JM, Ribot CA. Relative influence of age and menopause on total and regional body composition changes in postmenopausal women. Am J Obstet Gynecol. 1996;175:1594-1600.

11. Carr MC. The emergence of the metabolic syndrome with menopause. J Clin Endocrinol Metab. 2003;88:2404-2411.

12. Evans EM, Van Pelt RE, Binder EF, Williams DB, Ehsani AA, Kohrt WM. Effects of HRT and exercise training on insulin action, glucose tolerance, and body composition in older women. $J \mathrm{Appl}$ Physiol. 2001;90:2033-2040.

13. Gohlke-Barwolf C. Coronary artery disease - is menopause a risk factor? Basic Res Cardiol. 2000;95 Suppl 1:I77-I83.

14. Kuller LH, Simkin-Silverman LR, Wing RR, Meilahn EN, Ives DG Women's Healthy Lifestyle Project: a randomized clinical trial: results at 54 months. Circulation. 2001;103:32-37.

15. Simkin-Silverman L, Wing RR, Hansen DH, et al. Prevention of cardiovascular risk factor elevations in healthy premenopausal women. Prev Med. 1995;24:509-517.

16. Wyss JM, Carlson SH. Effects of hormone replacement therapy on the sympathetic nervous system and blood pressure. Curr Hypertens Rep. 2003;5:241-246.

17. Cho GJ, Lee JH, Park HT, et al. Postmenopausal status according to years since menopause as an independent risk factor for the metabolic syndrome. Menopause. 2008;15:524-529.

18. Asikainen TM, Suni JH, Pasanen ME, et al. Effect of brisk walking in 1 or 2 daily bouts and moderate resistance training on lower-extremity muscle strength, balance, and walking performance in women who recently went through menopause: a randomized, controlled trial. Phys Ther. 2006;86:912-923.

19. Gami AS, Witt BJ, Howard DE, et al. Metabolic syndrome and risk of incident cardiovascular events and death: a systematic review and meta-analysis of longitudinal studies. J Am Coll Cardiol. 2007;49: 403-414.

20. Hunter GR, McCarthy JP, Bamman MM. Effects of resistance training on older adults. Sports Med. 2004;34:329-348.

21. Lakka HM, Laaksonen DE, Lakka TA, et al. The metabolic syndrome and total and cardiovascular disease mortality in middle-aged men. JAMA. 2002;288:2709-2716.

22. Strasser B, Siebert U, Schobersberger W. Resistance training in the treatment of the metabolic syndrome: a systematic review and meta-analysis of the effect of resistance training on metabolic clustering in patients with abnormal glucose metabolism. Sports Med. 2010;40:397-415.

23. Haskell WL, Lee IM, Pate RR, et al. Physical activity and public health: updated recommendation for adults from the American College of Sports Medicine and the American Heart Association. Med Sci Sports Exerc. 2007;39:1423-1434.

24. Park MS, Chung SY, Chang Y, Kim K. Physical activity and physical fitness as predictors of all-cause mortality in Korean men. J Korean Med Sci. 2009;24:13-19.

25. Johnson JL, Slentz CA, Houmard JA, et al. Exercise training amount and intensity effects on metabolic syndrome (from Studies of a Targeted Risk Reduction Intervention through Defined Exercise). Am J Cardiol. 2007;100:1759-1766.

26. Katzmarzyk PT, Leon AS, Wilmore JH, et al. Targeting the metabolic syndrome with exercise: evidence from the HERITAGE Family Study. Med Sci Sports Exerc. 2003;35:1703-1709.

27. American College of Sports Medicine. American College of Sports Medicine position stand. Progression models in resistance training for healthy adults. Med Sci Sports Exerc. 2009;41:687-708.

28. Bateman LA, Slentz CA, Willis LH, et al. Comparison of aerobic versus resistance exercise training effects on metabolic syndrome (from the Studies of a Targeted Risk Reduction Intervention Through Defined Exercise - STRRIDE-AT/RT). Am J Cardiol. 2011;108: 838-844.
29. Libardi CA, Souza GV, Gáspari AF, et al. Effects of concurrent training on interleukin-6, tumour necrosis factor-alpha and C-reactive protein in middle-aged men. J Sports Sci. 2011;29:1573-1581.

30. Orsatti FL, Nahas EA, Maesta N, Nahas-Neto J, Burini RC. Plasma hormones, muscle mass and strength in resistance-trained postmenopausal women. Maturitas. 2008;59:394-404.

31. Augusto Libardi C, Bonganha V, Soares Conceição M, et al. The periodized resistance training promotes similar changes in lipid profile in middle-aged men and women. J Sports Med Phys Fitness. 2012;52: 286-292.

32. Treuth MS, Hunter GR, Kekes-Szabo T, Weinsier RL, Goran MI, Berland L. Reduction in intra-abdominal adipose tissue after strength training in older women. J Appl Physiol. 1995;78:1425-1431.

33. Florindo AA, et al. [Validation and reliability of the Baecke questionnaire for the evaluation of habitual physical activity in adult men] Validação e reprodutibilidade do questionário de Baecke de avaliação da atividade física habitual em homens adultos. Rev Bras Med Esporte. 2003;9:121-128. Portuguese.

34. Jackson AS, Pollock ML, Ward A. Generalized equations for predicting body density of women. Med Sci Sports Exerc. 1980;12:175-181.

35. Siri WE. Body composition from fluid spaces and density: analysis of methods. 1961. Nutrition. 1993;9:480-491.

36. Stone MH, O'Bryant H, Garhammer J. A hypothetical model for strength training. J Sports Med Phys Fitness. 1981;21:342-351.

37. Campos GE, Luecke TJ, Wendeln HK, et al. Muscular adaptations in response to three different resistance-training regimens: specificity of repetition maximum training zones. Eur J Appl Physiol. 2002; 88:50-60.

38. Jurca R, Lamonte MJ, Barlow CE, Kampert JB, Church TS, Blair SN. Association of muscular strength with incidence of metabolic syndrome in men. Med Sci Sports Exerc. 2005;37:1849-1855.

39. Wijndaele K, Duvigneaud N, Matton L, et al. Muscular strength, aerobic fitness, and metabolic syndrome risk in Flemish adults. Med Sci Sports Exerc. 2007;39:233-240.

40. Sternfeld B, Bhat AK, Wang H, Sharp T, Quesenberry CP Jr. Menopause, physical activity, and body composition/fat distribution in midlife women. Med Sci Sports Exerc. 2005;37:1195-1202.

41. Johnston AP, De Lisio M, Parise G. Resistance training, sarcopenia, and the mitochondrial theory of aging. Appl Physiol Nutr Metab. 2008;33: 191-199.

42. Heymsfield SB, McManus C, Smith J, Stevens V, Nixon DW. Anthropometric measurement of muscle mass: revised equations for calculating bone-free arm muscle area. Am J Clin Nutr. 1982;36:680-690.

43. Knapik JJ, Staab JS, Harman EA. Validity of an anthropometric estimate of thigh muscle cross-sectional area. Med Sci Sports Exerc. 1996;28:1523-1530.

44. Holten MK, Zacho M, Gaster M, Juel C, Wojtaszewski JF, Dela F. Strength training increases insulin-mediated glucose uptake, GLUT4 content, and insulin signaling in skeletal muscle in patients with type 2 diabetes. Diabetes. 2004;53:294-305.

45. Pattyn N, Cornelissen VA, Eshghi SR, Vanhees L. The effect of exercise on the cardiovascular risk factors constituting the metabolic syndrome: a meta-analysis of controlled trials. Sports Med. 2013;43: 121-133.

46. Potteiger JA, Claytor RP, Hulver MW, et al. Resistance exercise and aerobic exercise when paired with dietary energy restriction both reduce the clinical components of metabolic syndrome in previously physically inactive males. Eur J Appl Physiol. 2012;112:2035-2044. 


\section{Publish your work in this journal}

Clinical Interventions in Aging is an international, peer-reviewed journal focusing on evidence-based reports on the value or lack thereof of treatments intended to prevent or delay the onset of maladaptive correlates of aging in human beings. This journal is indexed on PubMed Central, MedLine, the American Chemical Society's 'Chemical Abstracts

Service' (CAS), Scopus and the Elsevier Bibliographic databases. The manuscript management system is completely online and includes a very quick and fair peer-review system, which is all easy to use. Visit http://www.dovepress.com/testimonials.php to read real quotes from published authors.

Submit your manuscript here: http://www.dovepress.com/clinical-interventions-in-aging-journal 